\title{
Dynamic Simulation on Impact Test of Vehicle Wheel
}

\author{
Yuqing Zheng ${ }^{1, \text { a }}$, Bing $\mathrm{Li}^{2, \mathrm{~b}}$ and Zhenlin Wang ${ }^{3, \mathrm{c}}$ \\ 1. Information and engineering school, Huzhou Teachers College, Huzhou Zhejiang, 313000 \\ 2. School of Aeronautics and Astronautics, Zhejiang University, Hangzhou, Zhejiang 310017



Keywords: Dynamic simulation, Vehicle wheel, Impact test, Mass scaling.

\begin{abstract}
This paper establishes a numerical procedure to predict the aluminum wheel performance during the impact test. The dynamic finite element solver, Ansys-Lsdyna970, is used. In order to save the computation time, the striker is assigned with an initial velocity, which is equal to the velocity reached during the free-fall period upon release. Mass scaling method is also utilized to further reduce computational time. Equivalent plastic strain is used as the damage indicator to judge pass or fail for the dynamic impact test. The true stress-strain curve is obtained from a uni-axial tensile test of A356-T6 samples machined from a prototype wheel. Simulation results show that plastic deformation tends to be localized around spoke-to-hub junction area. Studies on a recent prototype wheel revealed good correlation between experimental results and numerical prediction.
\end{abstract}

\section{Introduction}

Impact performance is critical for wheels to ensure vehicle safety and drive quality. This procedure establishes the minimum performance requirements for wheels of the passenger car, SUV and light truck. A wheel is usually consisted by 5 regions, which are hub, spoke, rim, flange and window, as shown in Fig. 1. The wheel also needs to pass the multiple checks to ensure that it is not damaged prior to the test.

At the same time, the numerical simulations of wheel impact test are essential in the wheel development process, which can reduce design time, especially for prototype building or concept verification in the early product development phase. When it is appropriately carried out, nonlinear finite element analysis can help engineer to validate the wheel design. Therefore, physical impact tests can be reduced or even eliminated after establishing the correlation between numerical simulation and lab impact test.

\section{FEA Model}

According to the vehicle wheel impact test regulations defined in the SAE $\mathrm{J} 175^{[1]}$, considering partial impact energy absorbed by rubber tire, so the simplified FEA model is built and assembled by two parts, namely the wheel and the striker, as shown in Fig.2 and the striker mass is determined by Eq.1.

$$
D=0.6 \mathrm{~W}+180
$$

where $\mathrm{D}$ is the striker mass in $\mathrm{kg}$ and $\mathrm{W}$ is the maximum static wheel load specified by the wheel itself and/or vehicle manufacturer, also expressed in $\mathrm{kg}$. In order to enhance the safety of vehicle driving in reality, so the vehicle manufacturer actively requires adding the test striker mass (D) up to $420 \mathrm{~kg}$.

The wheel is modeled as an elasto-plastical deformable body while the striker is simplified as a $420 \mathrm{~kg}$ rigid body. The load and boundary conditions are set in a way similar to those in actual impact test procedure in the SAE J175. All degrees of freedom of the nodes on the mounting surface of bolt holes are fully constrained. In addition, the striker is only given an initial vertical velocity, as it behaves in the impact test.

\section{Numerical Approaches}

To evaluate the wheel performance in the impact test, one failure criteria, no fracture occurrence in the centre member of the wheel, is defined by SAE J175. The true stress-true plastic strain tensile curve of A356-T6 aluminum is shown in the Fig.3. Based on the material tensile curve, the allowable maximum equivalent strain is set to be 0.035 as the criterion for impact simulation analysis to judge the wheel pass the test or not. The mechanical properties of the spoke are listed in Table 1. 

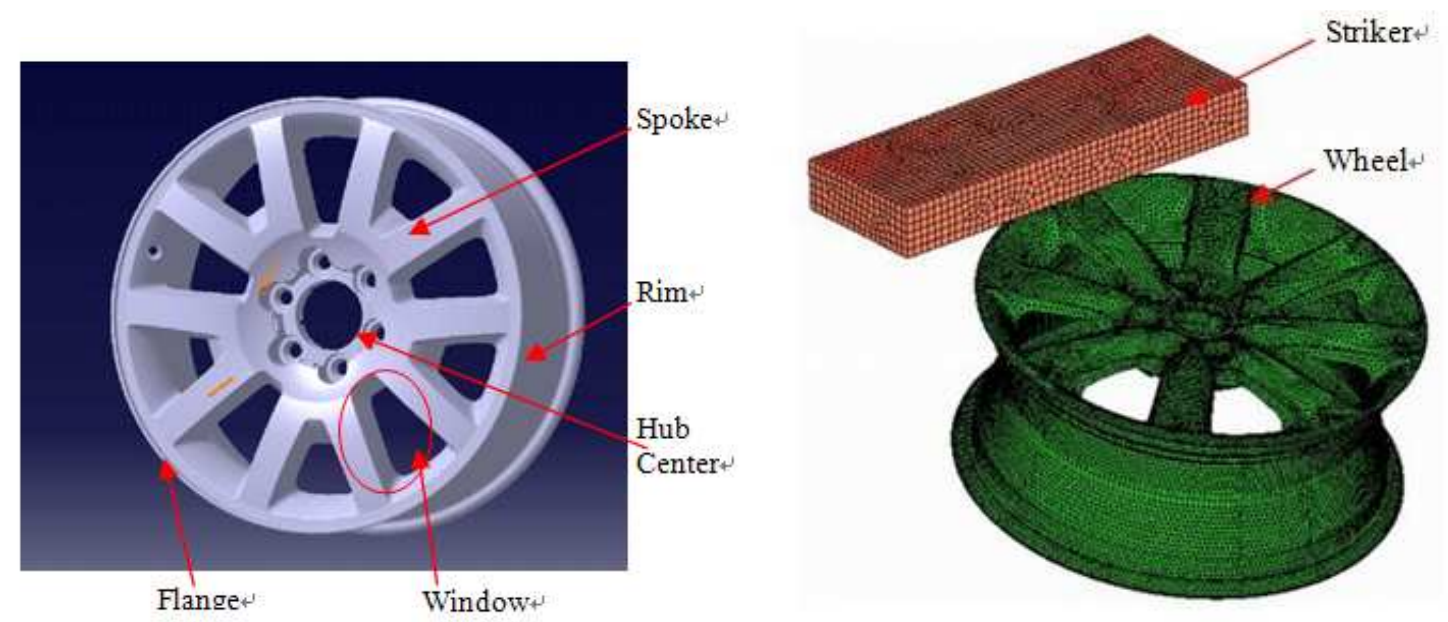

Fig. 1. Wheel Structure Terminology

Fig. 2. Finite Element Model for Wheel Impact

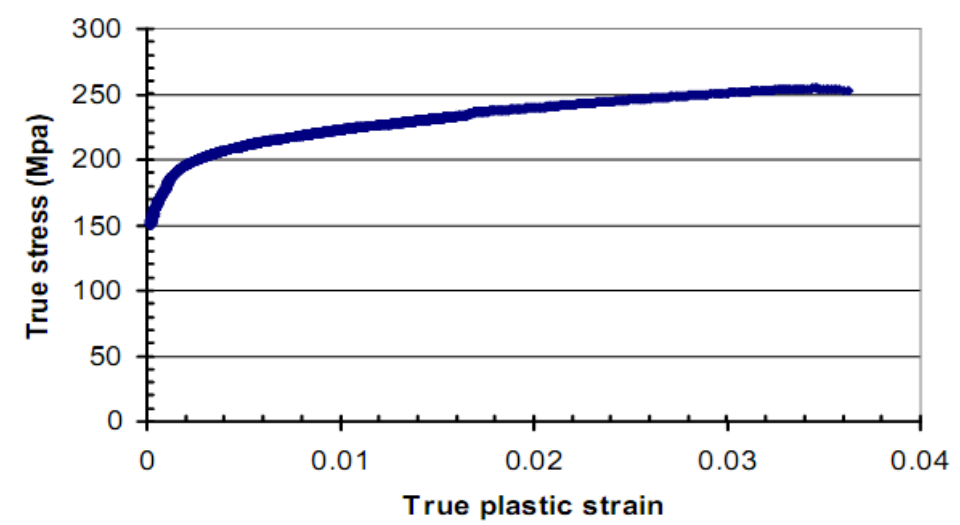

Fig. 3. True stress and true plastic strain curve of A356-T6 aluminum

Table 1 Mechanical properties of the spoke

\begin{tabular}{|l|c|c|c|c|}
\hline $\begin{array}{l}\text { Mechanics } \\
\text { Properties }\end{array}$ & Yield Strength & Tensile Strength & Elongation at Break & Young's Modulus \\
\cline { 2 - 5 } & $150(\mathrm{Mpa})$ & $255(\mathrm{Mpa})$ & $4 \%$ & $66(\mathrm{Gpa})$ \\
\hline
\end{tabular}

Since the tire will absorb partial kinetic energy from the dropping striker during impact testing, the general practice is to deduct a certain percentage of the striker's kinetic energy to compensate for the exclusion of tire in the simulation.Before the striker contacts with the tire, the total kinetic energy of the striker is

$$
\mathrm{E}=\mathrm{mgH}
$$

where $\mathrm{m}$ is the striker mass, $\mathrm{H}$ is the free-fall distance. As a result of the energy loss due to the tire, the initial velocity $\mathrm{V}_{0}$ of the striker can be calculated as follows:

$$
v_{0}=\sqrt{\frac{2 m g H(1-\eta)}{m}}=\sqrt{2 g H(1-\eta)}
$$

Table 2 Initial velocity with different energy reduction percentage

\begin{tabular}{|c|l|l|l|l|l|}
\hline$\eta \mathbf{( \% )}$ & 10 & 15 & 20 & 25 & 30 \\
\hline $\left.\mathbf{v}_{\mathbf{0}} \mathbf{( m m} / \mathbf{m s}\right)$ & 2.014 & 1.957 & 1.899 & 1.839 & 1.776 \\
\hline
\end{tabular}

where $\eta$ is the energy reduction percentage due to tire deformation during the impact test and $\mathbf{v}_{0}$ is the initial velocity of striker just before it impacts the wheel. 5 different initial velocities for striker are listed in Table 2 with different deductions to count for rubber tire deformation. Previous studies showed that $20 \%$ energy loss is considered as a good estimate ${ }^{[2,3]}$. Therefore, the $20 \%$ reduction of the striker's kinetic energy is used in the dynamic simulation analysis of this vehicle wheel. 


\section{Simulation Results}

In this section, effects of mass scaling factor and impact location on overall wheel deformation will be discussed in sequence.

Effect of Mass Scaling Value. In this section, the striker impacts the end of spoke. In order to reduce computational time, mass scaling method is utilized in Ansys-Lsdyna970 ${ }^{[4,5]}$. By artificially setting the mass scaling value DT2MS negative in the '*Control_Timestep' card, TSSFAC* $|D T 2 M S|$ is the minimum time step (as required by stability criterion). Therefore, less time will be needed to complete the simulation analysis ${ }^{[6]}$.

Plastic strain contours and computation time with 2 different mass scaling values $(-5 e-4$ and -8e-4) are illustrated in Fig.4. As expected, computation time can be dramatically reduced from 5 hours to 2 hours when the mass scaling factor is $-8 \mathrm{e}-4$. When the mass scaling value is $-5 \mathrm{e}-4$, the result is almost the same as the $-8 \mathrm{e}-4$ 's. The total mass of the FEA model is added by $2.1 \%$, and computation time is reduced by $60 \%$ with low accuracy loss. It is proved that the simulation result with the mass varying range $<5 \%$ is acceptable ${ }^{[7]}$.

It is concluded that balance between accuracy and computational cost can be achieved by using appropriate mass scaling technique.

Effect of Impact Location. As stated in SAE J175, multiple locations on the rim should be tested to ensure the integrity of the wheel. Fig. 5 lists the simulation results of two typical load cases: impact at the spoke end (Case A) and impact at the middle of the window (Case B). For Case A, plastic deformation is concentrated on the spoke that is directly impacted on and the spoke-to-hub junction area, while for Case B plastic strain is mainly distributed across two adjacent spokes. The max strain is decreased from 0.01406 in Case A to 0.01398 in Case B. Both results have plastic strain that is less than the ultimate strain of A356. Therefore, this wheel can pass the impact test with good safety margin. Fig. 5 also indicates that the impact at location A is the most critical case for impact test.

Correlation between Test and Simulation. The above mentioned approach is applied to predict the impact performance of a recently designed wheel. The original wheel prototype did not pass the impact test in the lab and cracks were found on the spoke after the impact test. The location of the crack matched with CAE prediction. After modifying the wheel structure design, dynamic simulation was carried out and no server plastic deformation was found on the spoke. Later physical test results did not found any failure after impact test in the lab and the new structure design was therefore released for mass production.

\section{Conclusions}

1) A finite element model of aluminum wheel for impact testing is developed. It includes elastoplastic material behaviors (Mat24) and considers the kinetic energy loss due to the tire deformation in the impact test. Failure criterion is based on the equivalent plastic strain at break point that can be measured from a uniaxial tensile test in the lab.

2) Mass scaling method can dramatically reduce the computational time with little sacrifice of accuracy, which can accelerate the product design and development process.

3) The impact location will affect the plastic strain distribution and magnitude. It is recommended to perform simulation and test for impact on spoke only because that is the worst case.

4) Dynamic simulation can effectively predict the impact performance of the aluminum wheel after coming out the good correlation between impact test and simulation. 




DT2MS $=-5 \mathrm{e}-4$, Computational time $=5 \mathrm{~h}$

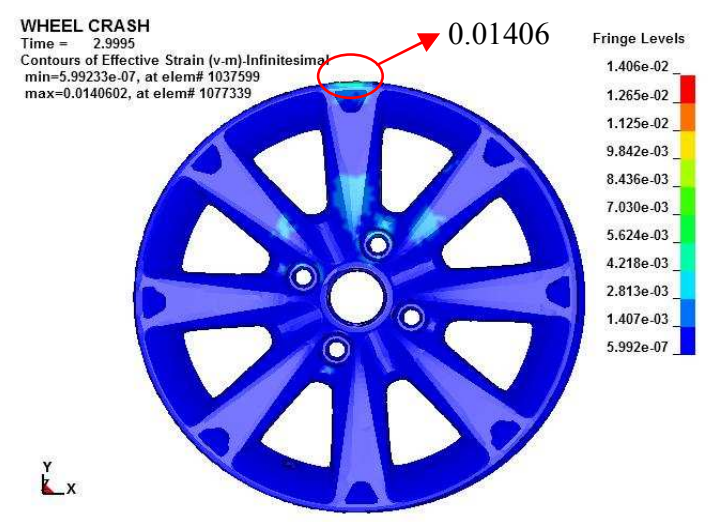

DT2MS $=-8 \mathrm{e}-4$, Computational time $=2 \mathrm{~h}$

Fig. 4. Analysis results with different mass scaling values

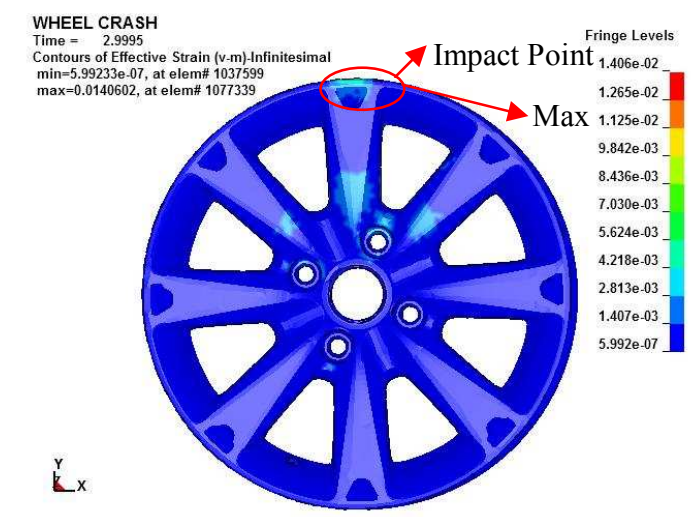

Case A: Max Plastic Strain $=0.01406$



Case B: Max Plastic Strain $=0.01398$

Fig. 5. Different impact locations

\section{References}

[1] Society of Automotive Engineers, "Wheels - Impact Test Procedure - Road Vehicles", J, SAE J175, SAE Handbook, Volume 4, 400 Commonwealth Drive, Warrendale, PA, USA, 1996.

[2] Hyung-Joo Hong, Jason Kline, Robert Geisler and Prasad Kodali, "Dynamic Front Wheel Curb Impact Study", J, SAE Paper no. 2005-01-1401, 2005 SAE World Congress, Detroit, MI, USA, 2005.

[3] Robert Shang, William Altenhof, and Herry Hu, "Kinetic Energy Compensation of Tire Absence in Numerical Modeling of Wheel Impact Testing", J, SAE Paper no. 2005-01-1825, 2005 SAE World Congress, Detroit, MI, USA, 2005.

[4] Ls-Dyna keyword user's manual version 970. Livermore Software Technology Corporation. 2003.

[5] Hallquist, J., LS-DYNA3D Theoretical Manual. Livermore Software Technology Corporation (LSTC): Livermore, 2006.

[6] Albert Satorres, "Bending simulation of High strength steel by finite elements", Master's thesis, University of Oulu, 2005.

[7] Ahmed Elmarakbi,Khaled Sennah, Magdy Samaan. "Crashworthiness of Motor Vehicle and Traffic Light Pole in Frontal Collisions", J, Journal of Transportation Engineering, 2006. 9:722 -733 . 